\title{
Phonon Dispersion Relation of Carbon Nanotube
}

\author{
A. BAHARI* AND M. Amiri \\ Department of Physics, Faculty of Basic Science \\ University of Mazandaran P.O. Box 47416, 476 Babolsar, Iran
}

(Received October 5, 2008)

Some researchers obtained the phonon dispersion relation of carbon nanotube by considering harmonic oscillation of carbon atoms. Recently, we could find an alternative method for determining carbon nanotube band energy by modifying orthogonalized-plane-wave method and combining it with tight binding method. Here, we did some calculations based on these methods and solved the dynamical matrix of carbon nanotube by referring the above methods and using force constant model. Some necessary adjustments are made in order to take into account the effect of the second nearest neighbor atoms which are not harmonic vibrations.

PACS numbers: 04.62.+v, 98.80.Cq, 12.10.Dm

\section{Introduction}

Carbon nanotubes (CNTs) become an important technology because of their outstanding performance in wide engineering applications. It has been called the wonder material of the 21st century, the building blocks for the future of electronics and the replacement for silicon circuits $[1,2]$. These materials have been hailed for their material properties and the applications that these properties promise. Their high electrical and thermal conductivity, remarkable strength, elasticity and anisotropic shape suggest numerous possibilities of nanoscale biological probes and structural elements in many electronic devices [3-5].

A CNT, simply, is the honeycomb lattice of a graphene sheet rolled into a cylinder. Graphene is also the name commonly associated with a single layer of graphite in two dimensions. The graphene sheet lattice structure is not a Bravais lattice by itself, but can be regarded as an underlying square Bravais lattice with a two-atom basis.

There are typical covalent bonds between a carbon atom in a graphene and its neighboring atoms, namely, each carbon atom has three neighboring atoms to which it is connected.

The rule explored in the present work is based on vibration of the nearest neighbor atoms which would yield a coverage final state of deformation under given loads or displacements at specific sites. In fact, as we show in our other paper [6], the idea is based on orthogonalized-plane-wave and tight binding methods which are placed in the force constant model. We could thus modify the phonon dispersion relation and show that the second nearest neighboring atoms vibrate with the different amplitude, direction and phase from those for the first nearest neighboring atoms.

\section{Calculations and discussion}

As mentioned previously, graphene is a two-dimensional sheet consisting of connected carbon atoms

\footnotetext{
* corresponding author; e-mail: a.bahari@umz.ac.ir
}

in hexagons like the benzene molecule. The basis of a graphene sheet consists of two atoms named "a" and "b", see Fig. 1. When considering only nearest neighbor interaction between e.g. the "a" atom and the nearest neighbors, which are three "b" atoms, the angle between each "b" atom is equally spaced with $120^{\circ}$. When folding the graphene sheet into a single walled carbon nanotube (SWCNT) it is possible to use the concepts and calculations from graphene sheets [5].

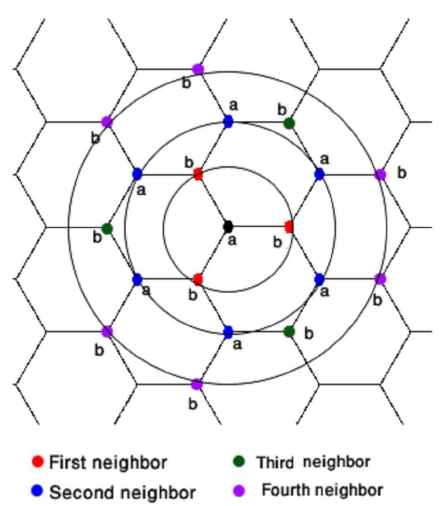

Fig. 1. Neighbor atoms of a graphitic plane up to 4 th nearest neighbors for an "a" atom. Circles connecting the same neighbor atoms are for guides to the eye.

Indeed, the calculations are performed on graphene sheets, which is a good approximation if the radius of the CNT is much greater than the distance between two neighboring nuclei of the carbon atoms in the CNT. As we know from the literature like $[3,6,7]$, the diameter of most CNTs is between $0.7 \mathrm{~nm}$ and $2 \mathrm{~nm}$, whereas the distance between the nuclei of the atoms is $1.42 \AA$.

Therefore, the force constant-phonon dispersion calculations in graphene are valid on CNTs as well, where the nearest neighbor interaction among pure $\pi$ orbitals of the carbon atoms in the graphene sheet is dominating with respect to $\sigma$ orbitals.

For this purpose, we pick out one particular atom to study and consider all of the other nearest neighbor's atoms on this atom. This arbitrary carbon atom 
of graphene can vibrate (move) freely while its nearest neighbor atoms are located at their positions without any displacement and movement. In this case, we assume that the tube cut open along the direction of its axis and the atoms lay out as a planner sheet. This yielded a 2D automat grid with only $x-y$ coordinates required to define the system state. Let us keep in mind that the most stable location of our arbitrary atom can be found by minimizing the total bonding energy.

Although there are both bonding and antibonding potentials in the graphene sheet [5], we neglect antibonding potential in the present work. Bonding potential includes harmonic and More's potentials $[8,9]$ in that

$$
\begin{aligned}
U & =U_{\text {bond }}+U_{\mathrm{LJ}}, \\
U & =D\left[\left(1-\mathrm{e}^{-\beta\left(r-r_{0}\right)}\right)^{2}-1\right] \\
& +\frac{1}{2} K\left(\theta-\theta_{0}\right)^{2}\left[1+K^{\prime}\left(\theta-\theta_{0}\right)^{4}\right] .
\end{aligned}
$$

$r_{0}=1.42 \AA, D=6.03 \times 10^{-19} \mathrm{~N} \mathrm{~m}, \beta=2.65 \times 10^{10} \mathrm{~m}^{-1}$, $\theta_{0}=2.094 \mathrm{rad}, K=9 \times 10^{-19} \mathrm{~N} \mathrm{~m} / \mathrm{rad}^{2}, K^{\prime}=$ $0.754 \mathrm{rad}^{-4}$, where the force corresponding to the above potentials can be determined by

$$
\boldsymbol{F}=\nabla U \text {. }
$$

Here we concentrate on the "velocity Verlet" algorithm, which may be written $[10,11]$ as

$$
\begin{aligned}
& p_{i}\left(t+\frac{1}{2} \delta t\right)=p_{i}(t)+\frac{1}{2} \delta t f_{i}(t), \\
& r_{i}(t+\delta t)=r_{i}(t)+\frac{\delta t p_{i}\left(t+\frac{1}{2} \delta t\right)}{m_{i}}, \\
& p_{i}(t+\delta t)=p_{i}\left(t+\frac{1}{2} \delta t\right)+\frac{1}{2} \delta t f_{i}(t+\delta t) .
\end{aligned}
$$

After step (4b), a force evaluation is carried out, to give $f_{i}(t+\delta t)$ for step $(4 \mathrm{c})$. This scheme advances the coordinates and moments over a time step $\delta t$.

Figure 2 shows the displacement of arbitrary atom which is plotted based on "velocity Verlet" algorithm. It represents harmonic vibrations with the same amplitude, direction and phase, where only arbitrary atom is allowed to move and vibrate.

If we consider the effect of just first nearest neighbor atoms on our arbitary atom, the behaviour of atom is completely different from that second nearest neighbor atoms and so on, as shown in Fig. 3. By comparing Figs. 2 and 3, we can see different behavior of carbon atoms due to vibrations of nearest neighbor atoms. Figure 2 confirms the harmonic vibrations of atom and the phonon dispersion relation can thus be determined by getting the slope of phonon dispersion curvature. However, the first nearest neighbor atom's vibrations cause inharmonic vibrations of atoms which was not reported before.

Figure 3 shows three peaks with different amplitudes which iterated by passing vibration's time give the simple approximation for this behavior

$$
x(t) \propto[\cos (\omega t)+\cos (2 \omega t)+\cos (3 \omega t)] .
$$

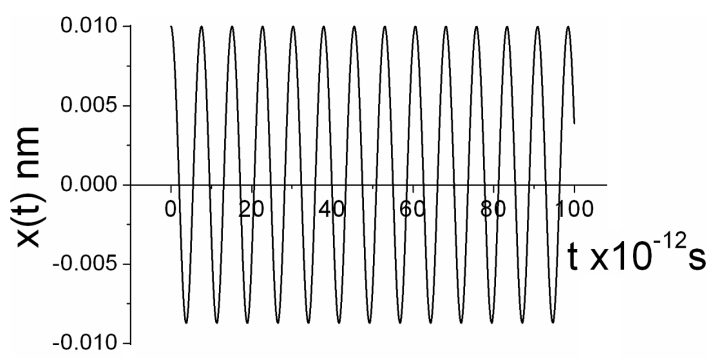

Fig. 2. Vibration of arbitrary atom. We consider the effect of just first nearest neighbor's atoms on the atom.

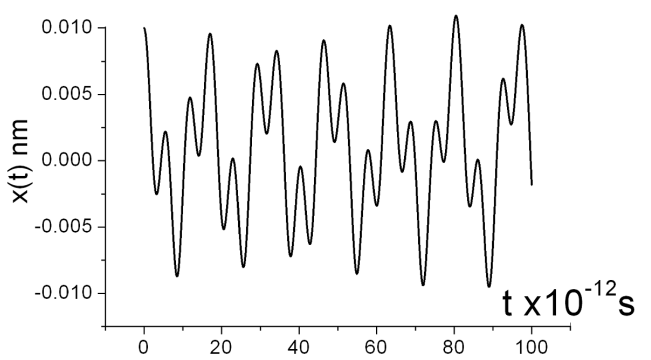

Fig. 3. Displacement of arbitrary atom. We consider the effect of just first nearest neighbor's atoms on the atom.

This function is shown in Fig. 4.

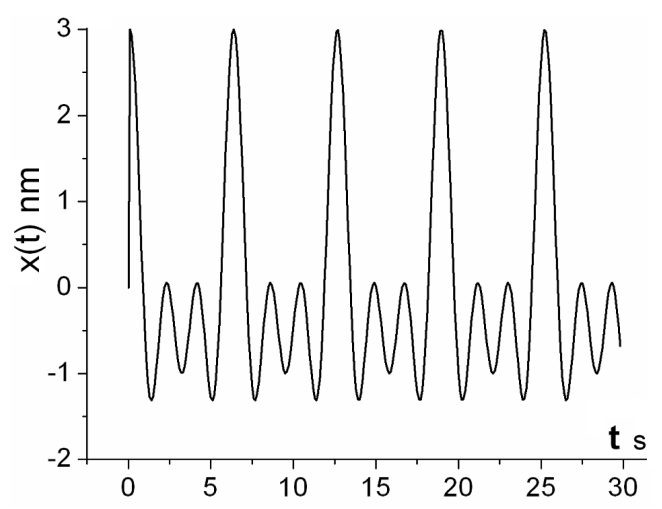

Fig. 4. The curvature of Eq. (5). We propose this displacement instead of Fig. 2.

In order to determine phonon dispersion relation we consider the sum of the forces on the $i$-th atom, $F_{i}$, for $N$ atoms in the unit cell as follows:

$$
\boldsymbol{F}_{i}=\sum_{j} K^{i j}\left[\boldsymbol{u}_{j}\left(\boldsymbol{R}_{j}\right)-\boldsymbol{u}_{i}\left(\boldsymbol{R}_{i}\right)\right], i=1, \ldots, N,
$$

where $u_{i}\left(R_{i}\right)$ and $R_{i}=\left(x_{i}, y_{i}, z_{i}\right)$ are the displacement and position of the $i$-th atom, respectively. The sum over $j$ is taken up to the nearest neighbor of the $i$-th atom and $K^{i j}$ is a $3 \times 3$ force constant matrix between the $i$-th and the $j$-th atom. The equation of motion is 


$$
\begin{gathered}
M_{i} \ddot{u}_{i}\left(\boldsymbol{R}_{i}\right)=\sum_{j} K^{i j}\left[u_{j}\left(\boldsymbol{R}_{j}\right)-u_{i}\left(\boldsymbol{R}_{j}\right)\right], \\
i=1, \ldots, N,
\end{gathered}
$$

where $M_{i}$ is the mass of the $i$-th atom. Assuming the time dependence of all $u_{i}\left(R_{i}\right)$ as the form of Eq. (5) $u\left(R_{i}\right)$ can be found by using Fourier transform as below

$$
\begin{aligned}
& u_{i}\left(\boldsymbol{R}_{i}\right)=\frac{1}{\sqrt{N_{a}}} \sum_{q^{\prime}} \mathrm{e}^{-\mathrm{i}\left(\boldsymbol{q}^{\prime} \cdot \boldsymbol{R}_{i}\right)} \\
& \quad \times\left(\mathrm{e}^{\mathrm{i} \omega t}+\mathrm{e}^{2 \mathrm{i} \omega t}+\mathrm{e}^{3 \mathrm{i} \omega t}\right) u_{i}\left(\boldsymbol{q}^{\prime}\right) .
\end{aligned}
$$

Substituting (8) into (7) and rearranging terms yields

$$
\begin{aligned}
& \sum_{j} K^{i j} \sum_{q^{\prime}} \mathrm{e}^{-\mathrm{i}\left(\boldsymbol{q}^{\prime} \cdot \boldsymbol{R}_{i}\right)}\left(\mathrm{e}^{\mathrm{i} \omega t}+\mathrm{e}^{2 \mathrm{i} \omega t}+\mathrm{e}^{3 \mathrm{i} \omega t}\right) u_{q}^{i}\left(\boldsymbol{q}^{\prime}\right) \\
& -M_{i} \omega^{2} I \sum_{q^{\prime}} \mathrm{e}^{-\mathrm{i}\left(\boldsymbol{q}^{\prime} \cdot \boldsymbol{R}_{i}\right)}\left(\mathrm{e}^{\mathrm{i} \omega t}+4 \mathrm{e}^{2 \mathrm{i} \omega t}+9 \mathrm{e}^{3 \mathrm{i} \omega t}\right) u_{q}^{i}\left(\boldsymbol{q}^{\prime}\right) \\
& =\sum_{j} K^{i j} \sum_{q^{\prime}} \mathrm{e}^{-\mathrm{i}\left(\boldsymbol{q}^{\prime} \cdot \boldsymbol{R}_{i}\right)}\left(\mathrm{e}^{\mathrm{i} \omega t}+\mathrm{e}^{2 \mathrm{i} \omega t}+\mathrm{e}^{3 \mathrm{i} \omega t}\right) u_{q}^{j}\left(\boldsymbol{q}^{\prime}\right) \cdot(9)
\end{aligned}
$$

By introducing a $3 \times 3$ identity matrix $I$, and multiplying

(9) with $\mathrm{e}^{\mathrm{i} q R_{i}}$ and summing over $R_{i}$, we have

$$
\begin{aligned}
& {\left[\sum_{j} K^{i j}\left(\mathrm{e}^{\mathrm{i} \omega t}+\mathrm{e}^{2 \mathrm{i} \omega t}+\mathrm{e}^{3 \mathrm{i} \omega t}\right)\right.} \\
& \left.-M_{i} \omega^{2}\left(\mathrm{e}^{\mathrm{i} \omega t}+4 \mathrm{e}^{2 \mathrm{i} \omega t}+9 \mathrm{e}^{3 \mathrm{i} \omega t}\right) I\right] u_{q}^{i} \\
& \quad-\sum_{j} K^{i j}\left(\mathrm{e}^{\mathrm{i} \omega t}+\mathrm{e}^{2 \mathrm{i} \omega t}+\mathrm{e}^{3 \mathrm{i} \omega t}\right) u_{q}^{j} \mathrm{e}^{\mathrm{i} \boldsymbol{q} \cdot \Delta \boldsymbol{R}_{i j}}=0,
\end{aligned}
$$

where $\boldsymbol{I}$ is a $3 \times 3$ unit matrix and $\Delta \boldsymbol{R}_{i j}=\boldsymbol{R}_{i}-\boldsymbol{R}_{j}$ is the relative coordinate of the $i$-th atom with respect to the $j$-th atom. The vibration of the $i$-th atom is coupled to that of the $j$-th atom through the $K^{i j}$ force constant matrix. We note that $j$ in Eq. (10) can be a site in the neighboring unit cell when the $i$-th atom is near the unit cell boundary. Then Eq. (10) can be formally written as follows by defining a $3 N \times 3 N$ dynamical matrix $D(q)$ :

$$
D(\boldsymbol{q}) \boldsymbol{u}_{q}=0 .
$$

To obtain the eigenvalues $M_{i} \omega^{2}$ for $D(q)$ we solve the secular equation $\operatorname{det} D(q)=0$ for a given $q$ vector

$$
\operatorname{det}(D(q))=0 \text {. }
$$

It is convenient to divide the dynamical matrix $D(q)$ into small $3 \times 3$ matrices $D^{i j}(q)$ where we denote $D(q)$ by $\left\{D^{i j}(q)\right\}$, and from Eq. (10) it follows:

$$
\begin{aligned}
& D^{i j}(q)=\left[\sum_{j^{\prime \prime}} K^{i j^{\prime \prime}}\left(\mathrm{e}^{\mathrm{i} \omega t}+\mathrm{e}^{2 \mathrm{i} \omega t}+\mathrm{e}^{3 \mathrm{i} \omega t}\right)\right. \\
& \left.-M_{i} \omega^{2}\left(\mathrm{e}^{\mathrm{i} \omega t}+4 \mathrm{e}^{2 \mathrm{i} \omega t}+9 \mathrm{e}^{3 \mathrm{i} \omega t}\right) I\right] \delta_{i j} \\
& -\sum_{j^{\prime}} K^{i j^{\prime}}\left(\mathrm{e}^{\mathrm{i} \omega t}+\mathrm{e}^{2 \mathrm{i} \omega t}+\mathrm{e}^{3 \mathrm{i} \omega t}\right) \mathrm{e}^{\mathrm{i} \boldsymbol{q} \cdot \Delta \boldsymbol{R}_{i j^{\prime}}}=0 .
\end{aligned}
$$

The dynamical matrix $D$ for two-dimensional graphite is written in terms of the $3 \times 3$ matrices $D_{\text {aa }}, D_{\text {ab }}, D_{\text {ba }}$ and $D_{\mathrm{bb}}$ for the "a" and "b" atoms within the unit cell as shown in Fig. 1

$$
D(\boldsymbol{q})=\left[\begin{array}{cc}
D_{\mathrm{aa}} & D_{\mathrm{ab}} \\
D_{\mathrm{ba}} & D_{\mathrm{bb}}
\end{array}\right] .
$$

When we consider an "a" atom, the three nearest-neighbor atoms (see Fig. 1) are " $b_{1}$ ", "b $b_{2}$ " and " $b_{3}$ " whose contributions to $D$ are contained in $D_{\mathrm{ab}}$, while the six next-nearest-neighbor atoms are all "a" atoms, with contributions to $D$ that are contained in $D_{\text {aa }}$ and so on. To obtain $K^{i j}$, we consider the force constant between an "a" atom and a nearest-neighbor "b1" atom on the $x$ axis as shown in Fig. 5.

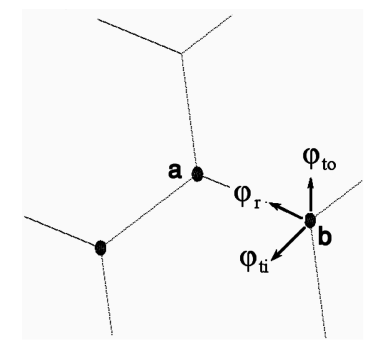

Fig. 5. Force constants between the "a" and "b" atoms on a graphene sheet.

The force constant tensor is given by

$$
K^{\mathrm{ab}_{1}}=\left[\begin{array}{ccc}
\varphi_{\mathrm{r}}^{1} & 0 & 0 \\
0 & \varphi_{\mathrm{ti}}^{1} & 0 \\
0 & 0 & \varphi_{\text {to }}^{1}
\end{array}\right],
$$

where $\varphi_{\mathrm{r}}^{n}, \varphi_{\mathrm{ti}}^{n}$, and $\varphi_{\mathrm{to}}^{n}$ represent the force constant parameters in the radial (bond-stretching), in-plane and out-of-plane tangential (bond-bending) direction of the $n$-th nearest neighbors, respectively.

The force constant matrices for the two other atoms are obtained by rotating the matrix in Eq. (15) according to the rules for a second-rank tensor

$$
K^{i j}=U^{-1}(\theta) K^{\mathrm{ab}_{1}} U(\theta),
$$

where $U$ is the rotation matrix for rotation around the $z$-axis, and $\theta$ is angle between $x$-axis and the line from one atom to another atom.

Furthermore, force constant parameters up to 4th nearest neighbor in unit of $10^{9} \mathrm{~N} / \mathrm{m}$ are determined experimentally by using inelastic neutron scattering or electron energy loss spectroscopy (EELS) techniques [5] are given in Table (see also Fig. 5).

\section{TABLE}

Force constant parameters up to 4 th nearest neighbor in unit of $10^{9} \mathrm{~N} / \mathrm{m}$.

\begin{tabular}{l|l|l}
\hline \hline Radial & $\begin{array}{l}\text { Tangential } \\
\text { in-plane }\end{array}$ & $\begin{array}{l}\text { Tangential } \\
\text { out-of-plane }\end{array}$ \\
\hline$\varphi_{\mathrm{r}}^{1}=36.5$ & $\varphi_{\mathrm{ti}}^{1}=24.5$ & $\varphi_{\mathrm{to}}^{1}=9.82$ \\
$\varphi_{\mathrm{r}}^{2}=8.8$ & $\varphi_{\mathrm{ti}}^{2}=-3.23$ & $\varphi_{\mathrm{to}}^{2}=-0.4$ \\
$\varphi_{\mathrm{r}}^{3}=3.00$ & $\varphi_{\mathrm{ti}}^{3}=-5.25$ & $\varphi_{\mathrm{to}}^{3}=0.15$ \\
$\varphi_{\mathrm{r}}^{4}=-1.92$ & $\varphi_{\mathrm{ti}}^{4}=2.29$ & $\varphi_{\mathrm{to}}^{4}=-0.58$
\end{tabular}


Figure 1 shows the number and kind of neighboring atoms, where each "a" ("b") atom has 6 (12) and 12 (6) nearest "a" ("b") and "b" ("a") atoms, respectively.

In Figs. 6 and 7 the phonon dispersion curves in two and three dimensions, respectively, of graphene sheet are shown by assuming the time dependence of all $u_{i}\left(R_{i}\right)$ as the form of $\mathrm{e}^{\mathrm{i} \omega t}$. We can see in Fig. 8 how the determinant of dynamical matrix of a two-dimensional graphene sheet as the form of Eq. (5) varies by passing vibrations time.

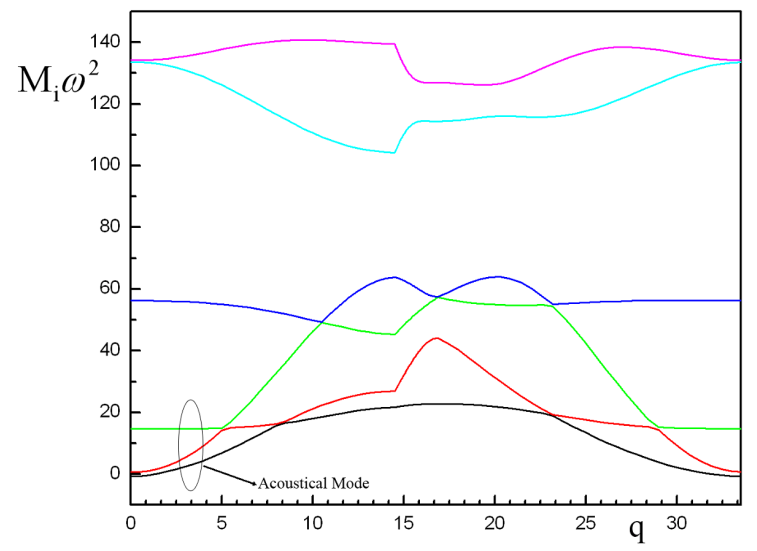

Fig. 6. The phonon dispersion curves plotted along to the axis of nanotube, for a $2 \mathrm{D}$ graphene sheet, using the set of force constants in Table in unit of $10^{9} \mathrm{~kg} \mathrm{~s}^{-2}$. There were assumed the same eigenfrequencies $\omega$ for all $u_{i}$ in Eq. (7). There are 6 phonon branches. Three lower branches are on the $\Gamma$ center called acoustic modes and the other branches are optical modes.

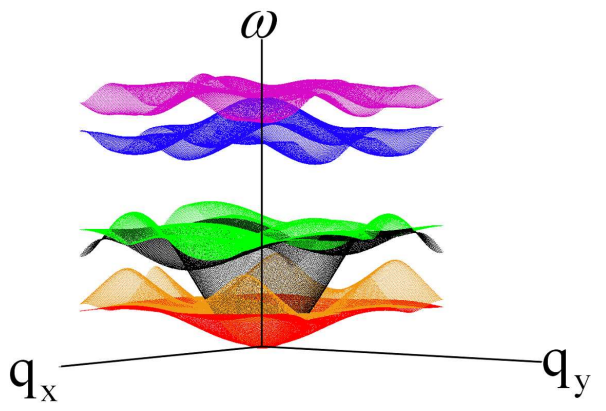

Fig. 7. 3D plot of the stable phonon dispersion in the first Brillouin zone.

Furthermore, our suggested Eq. (5) for time dependence of displacement vector causes an time oscillating behavior on phonon frequency. We estimate that the period time of phonon frequency is about $7.12 \mu \mathrm{s}$, based on above calculations.

\section{Conclusion}

The results of present work show harmonic oscillation of carbon atom in the CNT by considering only the first

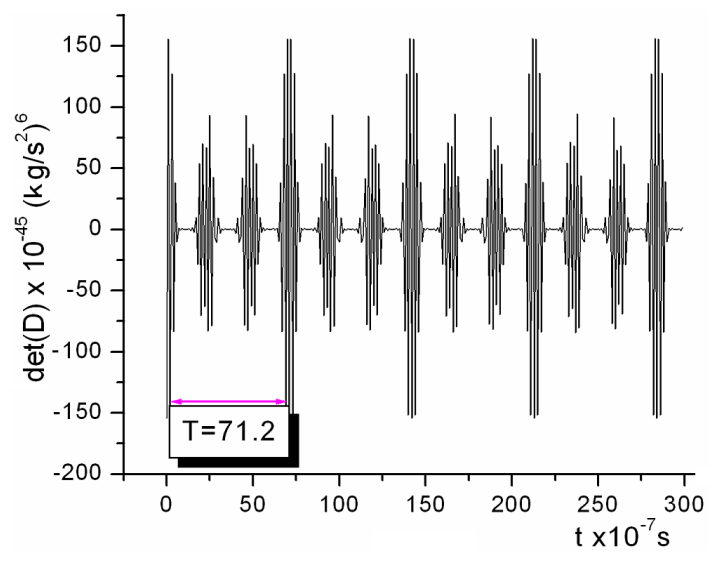

Fig. 8. The calculated determinant of dynamical matrix (Eq. (16)) from $t=0$ to $30 \mu$ s for $\omega=5$ at point of $(8,4)$ in the first the Brillouin zone. Our calculations show that determinant of dynamical matrix is a harmonic ocillation with a period time equal to $7.12 \mu \mathrm{s}$.

nearest neighbor atom's vibrations and anharmonic oscillation when the second (and above) nearest neighbor atoms are also taken into account. It means that the phonon dispersion relation of CNTs should be modified in accordance to what is shown in Fig. 8.

\section{Acknowledgments}

Partial support for this research received under a grant from university of Mazandaran, Iran.

\section{References}

[1] P. Avouris, IEEE Spectrum 1, 41 (2004).

[2] J. Ryoo, P. Hajela, J. Suhr, N. Korptkar, Adv. Eng. Software 38, 531 (2007).

[3] E. Schröder, P. Hyldgaard, Mater. Sci. Eng. C 23, 721 (2003).

[4] T.-S. Xia, L.F. Register, S.K. Banerjee, Phys. Rev. B 70, 045322 (2004).

[5] H. Raffi-Tabar, Phys. Rep. 390, 235 (2004).

[6] A. Bahari, Acta Phys. Pol. A 115, (2009).

[7] R. Satio, G. Dresselhaus, M.S. Dresselhaus, Physical Properties of Carbon Nanotubes, 1st ed., Imperial College Press, London 1998.

[8] J.M. Leamy, Int. J. Solids Struct. 44, 874 (2007).

[9] J. Che, T. Cagin, W. Goddard, Nanotechnology 10, 263 (1999).

[10] W.C. Swope, H.C. Andersen, P.H. Berens, K.R. Wilson, J. Chem. Phys. 76, 637 (1982).

[11] J.W. Kang, H.J. Hwang, J. Korean Phys. Soc. 46, 875 (2005). 